\title{
Down syndrome detection using modified adaboost algorithm
}

\author{
Vincy Devi V. K $\mathbf{K}^{\mathbf{1}}$, Rajesh $\mathbf{R}^{2}$ \\ ${ }^{1}$ Department of Computer Science, Bharathiar University, Coimbatore, India \\ ${ }^{2}$ Department of Computer Science, CHRIST (Deemed to be University), Bangalore, India
}

\begin{tabular}{l}
\hline \hline Article Info \\
\hline Article history: \\
Received Jun 14, 2020 \\
Revised Mar 1, 2021 \\
Accepted Mar 14, 2021 \\
\hline
\end{tabular}

Keywords:

AdaBoost algorithm

Backpropagation

Bayes classifier

Down syndrome

Facial points

\begin{abstract}
In human body genetic codes are stored in the genes. All of our inherited traits are associated with these genes and are grouped as structures generally called chromosomes. In typical cases, each cell consists of 23 pairs of chromosomes, out of which each parent contributes half. But if a person has a partial or full copy of chromosome 21, the situation is called Down syndrome. It results in intellectual disability, reading impairment, developmental delay, and other medical abnormalities. There is no specific treatment for Down syndrome. Thus, early detection and screening of this disability are the best styles for down syndrome prevention. In this work, recognition of Down syndrome utilizes a set of facial expression images. Solid geometric descriptor is employed for extracting the facial features from the image set. An AdaBoost method is practiced to gather the required data sets and for the categorization. The extracted information is then assigned and used to instruct the Neural Network using Backpropagation algorithm. This work recorded that the presented model meets the requirement with $98.67 \%$ accuracy.
\end{abstract}

This is an open access article under the CC BY-SA license.

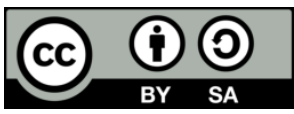

\section{Corresponding Author:}

Vincy Devi V. K.

Department of Computer Science

Bharathiar University

Coimbatore, India

Email: vincydevi15@gmail.com

\section{INTRODUCTION}

The bearing of a partial or full copy of chromosome 21 , commonly called Trisomy 21 results in a set of medical abnormalities called down syndrome (DS). An individual with DS may have individual and/or intellectual development issues [1]. Studies have shown that a soul suffering from DS exhibit diverse facial features [2]. The DS affected persons may also possess some characteristics that are a small chin, a flat nasal bridge, feeble muscle tone, and tilted eyes [3]. Diagnosis of DS may be executed earlier or after parturition. In the first case, bio-chemical evaluation and cytogenetic tests can be done for the diagnosis of DS. In the second case, the existence of a number of minor physical and medical malformations in addition to miniature ears protruded tongue, upslanting palpebral fissures, epicanthal fold, flat facial looks, and extremity variable points to the chance for DS [4]. For automated, computer-aided analysis of DS, the image process, and analysis can serve as an effective and powerful creature.

Loos et al. [5] studied the characteristic facial patterns using Gabor wavelet features for ten syndromes, excluding Down syndrome. Further studies [6] give a classification for 14 syndromes, but only $21 \%$ accuracy. Even so, that method failed to distinguish the syndromes from a tidy one. In improver, the method calls for manual labeling and so it was not a fully automated technique. Later, Saraydemir et al. [7] proposed a Gabor wavelet transform technique for the detection of DS and the technique possesses high accuracy. Burçin and Vasif developed a local binary pattern (LBP) based concept for the DS diagnosis. Here 
they used a template matching strategy. Still, these methods take some sort of manual pre-processing for the success of the diagnosis process.

Prior to birth, if the screening outcome reveals the chance for DS, a formal diagnosis can be established. For examining the modifications in specified anatomical features of the fetus, techniques like expanded alpha-fetoprotein (AFP) screening test, the nuchal translucency test, and supplementary ultrasound screens which are available [8]. Subjects were also claimed that the noses of children with DS may take in an under-developed nasal bone construction. But this deformation may not be identified during the early pregnancy scan [9]. These surveys confirm that the scientific reflection of fetal nasal bone contributes to the performance of the first-trimester screening for DS [10]. In machine learning (ML) boosting is an attitude that combines feeble and erroneous rules and in return create a precise forecast rule. The most widely used first practical boosting algorithm was the AdaBoost algorithm [11]. Adaboost is actually a machine-learning technique for face detection. In addition, the term boosted represents the classifiers at each stage, which is built of basic classifiers [12]. Many surveys were conducted for investigating the suitability of Adaboost in face recognition and the algorithm is found to be a better tool [13], [14]. In this algorithm, the face restructuring is done by moving the cascade detector across the image at various scales and locations. When compared with the number of regions scanned, a distinctive image has only a modest turn of face parts. However, in non-face regions, because of the untimely termination of the decision process, there are chances to get only a small number of stages of the cascade that are estimated at an average [15], [16]. Thus, the restraining factors in the speed of evaluation are the computational difficulty and the denial rates of the initial points.

Other challenges that may confront during the reorganization process are the non-availability of fine images, the garland of pictures and other image quality matters. So, for generating more accurate solutions, what is required is an efficient dataset. In this work, an enhanced AdaBoost algorithm is proposed for finding the different magnitude of DS. Various combinations of input parameters are suggested. It is noted that the proposed algorithm yields the finest results by using the military capabilities of the supported parameters. DS is recognized visually by seeing as the ultrasonogram image of the fetus for the occurrence of nasal bone (NB). Optical identification of NB may confuse the sonographers and leads to error because of the modest size of NB in the first trimester. Equally, it is visualized in the literature; the deficiency of nasal bone is a fundamental index to detect DS at the early phase of pregnancy. Speckle noise erodes the excellence of the ultrasound images. Therefore, the recognition of NB becomes very complex in the first trimester [17]. Figure 1 shows the Facial distinctiveness of a fetus used to analyze DS.
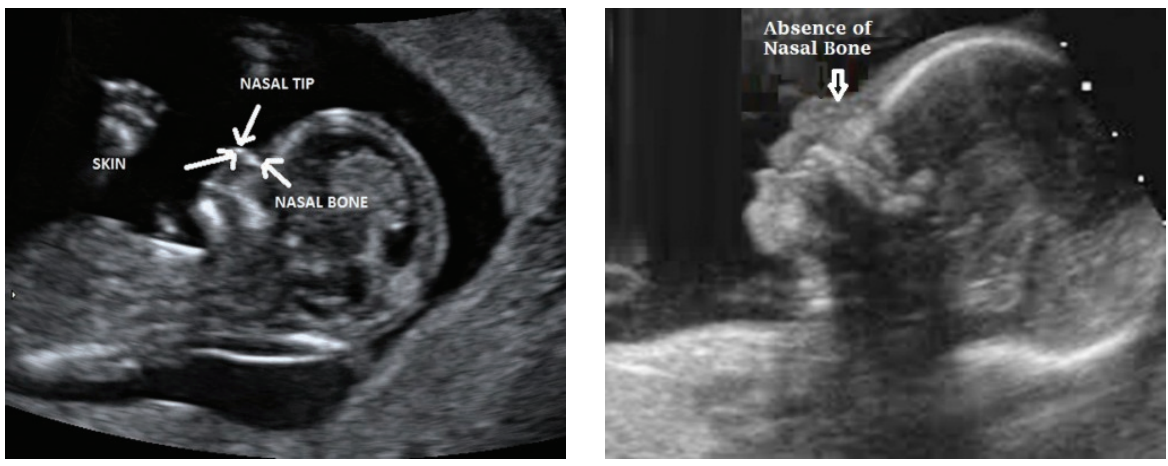

Figure 1. Facial distinctiveness of a fetus used to analyze down syndrome

This paper proposes an algorithm to detect the chance for DS through the analysis of fetus facial characters. Localization of facial points is done and the intercanthal distance is calculated. Classified the collected information into two based on the age. Using these calculations, the proposed algorithm detects the chance for DS. The remaining parts of the composition are arranged as follows. Part II presents the materials and methods, section 3 is the result and discussions and finally, section 4 concludes the study.

\section{MATERIALS AND METHODS}

\subsection{Adaptive boosting (AdaBoost) algorithm}

An AdaBoost algorithm is a tool to upgrade the accuracy of weak learning algorithms. It is a ML algorithm introduced by Freund and Schapire [17]. It can be implemented by running the weak learning 
algorithm multiple times, such that the data is slightly modified in each run and then combine the prepositions. It can be named as a meta-algorithm since it is utilized to increase the functioning of other scholars. In general, there are two types of classifiers in learning algorithms; strong and weak classifiers. A strong classification algorithm uses methods like artificial neural network (ANN) and support vector machine (SVM). However, a weak classification algorithm employs methods such as a bayesian network, and decision tree. AdaBoost classifier is shown in Figure 2. The AdaBoost algorithm is adaptive in nature. This is because if the previous classifier has misclassified any instances, and so those instances were restructured into the succeeding classifier. This algorithm is also really sensitive to disturbance. This algorithm starts by allocating the same weights to all occurrences in the training data. Then it forms a classifier using the learning algorithm for the data and the weights will get updated based on the classifier's response. The easy instances get low weights and high weights are given for hard cases. For the weighted data, a classifier is assigned in the following iteration. All these steps aim for a better classification. The instance weights are restructured based on the reaction of the new classifier. There are two probabilities, either the easier instance, may become easier or become harder. The second is, the harder instance becomes harder or easier. They are renormalized after the weight updates. As the last stair, the assumed value is estimated [18], [19].

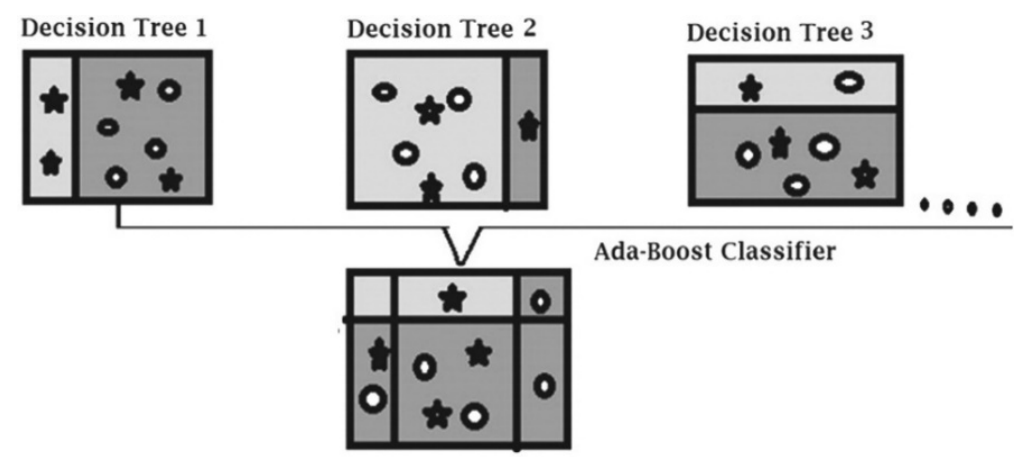

Figure 2. AdaBoost classifier

Algorithm

a) Start

b) Form training sample sets, initialize sample weights, and iterations. The weight

$$
w_{j i}=\frac{1}{m} ; \text { for } \mathrm{j}=1 \text { to } \mathrm{N} ; \mathrm{N} \text { is the number of iterations. }
$$

c) Calculate the normalized weights

$$
p_{j}(i)=\frac{w(i)}{\sum_{i}^{m} w(i)}
$$

d) Calculate the hypothesis $\left(h_{j}\right)$ value using Naïve Bayes probability

e) Calculate the error.

$$
e_{j}=\sum_{i} p_{j}(i)\left[h_{j}\left(x_{i} \neq y_{i}\right)\right]
$$

f) If eg $>1 / 2 ; \mathrm{N}=\mathrm{j}-1$; go to step 9

$$
\phi_{j}=\frac{e_{j}}{1-e_{j}}
$$

g) Calculate the weight coefficient,

h) Compute new weights using the coefficients 


$$
w_{j+1}(i)=w_{j}(i) \phi_{j}^{1-\left[h_{j}\left(x_{i}-y_{i}\right)\right]}
$$

i) Final hypothesis

$$
h_{o}(x)=\sum_{j=1}^{L}\left(\log \frac{1}{\phi_{h}}\right)\left[h_{j}(x=y)\right]
$$

j) Stop.

\subsection{Naïve baye's classifier}

In machine learning, a probabilistic classifier based on baye's [20] theorem is the naïve bayes classifiers. The provisional and minimal probabilities of two arbitrary events are related using bayes theorem. Generally, it is used to calculate the subsequent probabilities. These classifiers are independent feature models and learning such classifiers is simplified if the individual parameters are considered. When compared with other techniques, regardless of this naive hypothesis, this classifier is remarkably effective in practice, including for applications like a medical diagnosis, text classification, and systems performance administration. The conditional probability is presented equally,

$$
P\left(C_{k} \mid x\right)=\frac{P\left(x \mid C_{k}\right) P\left(C_{k}\right)}{\sum_{k^{\prime}} P\left(x \mid C_{k^{\prime}}\right) P\left(C_{k^{\prime}}\right)}
$$

The naïve bayes classifiers estimate that the value of the specific feature of a class is isolated from the value of any other feature. So,

$$
P\left(x \mid C_{k}\right)=\prod_{j=1}^{d} P\left(x^{j} \mid C_{k}\right)
$$

where, $\mathrm{C}=\{\mathrm{C} 1, \mathrm{C} 2, \ldots . . \mathrm{CK}\}$ is the set of class labels and $\mathrm{P}(\mathrm{x} \mid \mathrm{C})$ is conditional probability.

\subsection{Backpropagation neural network (BPNN)}

A neural network [21] is defined as a collection of associated input-output elements such that each connection has a weight linked with it. It is applied to make predictive models from big databases and they mimic the human neural system. For training feature sets extracted from the classifier, multi-layer BPNNs are used. Commonly, a multi-layer network consists of three layers of nerve cells. They are the input layer, a hidden layer, and output layer. The connections in BPNN are such that, each node in the input layer is connected to the nodes of the hidden layer. Also, hidden layer nodes are related to the output layer. There is no direct link between the nodes in an individual layer and with every connection; there should be an associated weighting factor [22]. At the time of training, the weights are modified using the BP algorithm and the process is called learning. In this study, the supervised learning method is adopted.

The ANN training parameters are presented in Table 1. The difference between target and actual response is termed as the system error. During the training process, this error is backpropagated to the preceding layer, i.e. the hidden layer. The error is then calculated for each element in this layer N. In the same manner, error at each node of a previously hidden layer that is $\mathrm{N}-1$ is also determined. By using this method to reduce the error to the expected level.

Table 1. ANN training parameters

\begin{tabular}{ccccc}
\hline No. of Inputs & No. of outputs & No. of Hidden Layer & No. of Hidden Neurons & No. of iterations \\
\hline 7 & 1 & 1 & 8 & 500 \\
Learning Rate & Momentum Rate & Activation Function & Training Method & Error \\
0.05 & 0.5 & Sigmoid & Backpropagation & 0.001 \\
\hline
\end{tabular}

\subsection{Proposed AdaBoost algorithm}

The Ada-Boost algorithm here used employes the naïve-bayes classifier as a weak learner. The initialization of training data and weights are based on the classifier. The weights are equal to the fraction of 
the total number of classifiers. Using the supervised learning method, these classifiers can be effectively developed. An advantage of the naive bayes classifier is that it requires only a little amount of training information for assessing the components needed for sorting. After each iteration new hypothesis $h_{j}$ is generated. Applying this hypothesis the weight and error are calculated and the system updates the weights based on the error value.

$$
w_{j+1}(i)=w_{j}(i) \beta_{j}^{1-\left[h_{j}\left(x_{i}-y_{i}\right)\right]}
$$

This procedure is repeated till the iterations are finished and the final value is computed utilizing the rule,

$$
h_{o}(x)=\sum_{j=1}^{L}\left(\log \frac{1}{\phi_{h}}\right)\left[h_{j}(x=y)\right]
$$

\subsection{Feature extraction}

In this proposed method, the facial landmarks are detected and then a geometric descriptor is built using it. The geometric representation is such that, it uses 2-D facial fiducial points. That is, two points P1 and $\mathrm{P} 2$ are chosen from the center of the eyebrows, for the glabella, one point- P3, four points P4, P5, P7, and P8 for the inner and outer corner of the eyes, one point P6 for the root of the nose, two points P10 and P11, for Alars sidewalls, one point P9 for the supratip, one point P12 for the columella, the points P14 and P15, for the mouth corners and two points P13 and P16 for the top and bottom of the upper lip and lower lip respectively. Figure 3 shows how the localization of the 16 facial points is done.

From the mentioned 16 points, fourteen distances are identified and taken out as shown in Figure 3. All these distances are normalized to the width of the face to guarantee that the characteristics are scaleinvariant The distances, say, d1,d2,d3, d6, d8, d10,d11,d12, and d14 symbolize the standard values of the two mirrored distances on both sides of the face. To calculate the distance d11, the meeting junctions of the line among the points on the top of the upper lip and bottom of the lower lip, and the line between the left and right corners of the mouth are used. Thus, the following characteristic vector has fourteen dimensions.

For recognizing DS, it is significant to consider the intercanthal space d4, i.e., space among the inside recesses of the eyes. An addition in the intercanthal distance called telecanthus is frequently observed in the people with DS. The presents of tiny palpebral fissures, which is the distance between the lateral and medial canthus of the optics is another common feature. This aspect is captured by space d3. People with DS commonly indicate the symptom of compressed nose and distances $\mathrm{d} 5$ to $\mathrm{d} 8$ stand for this particular feature. Lastly, the presence of a small mouth, which is a symptom of DS, is captured by distances d10 to d13.
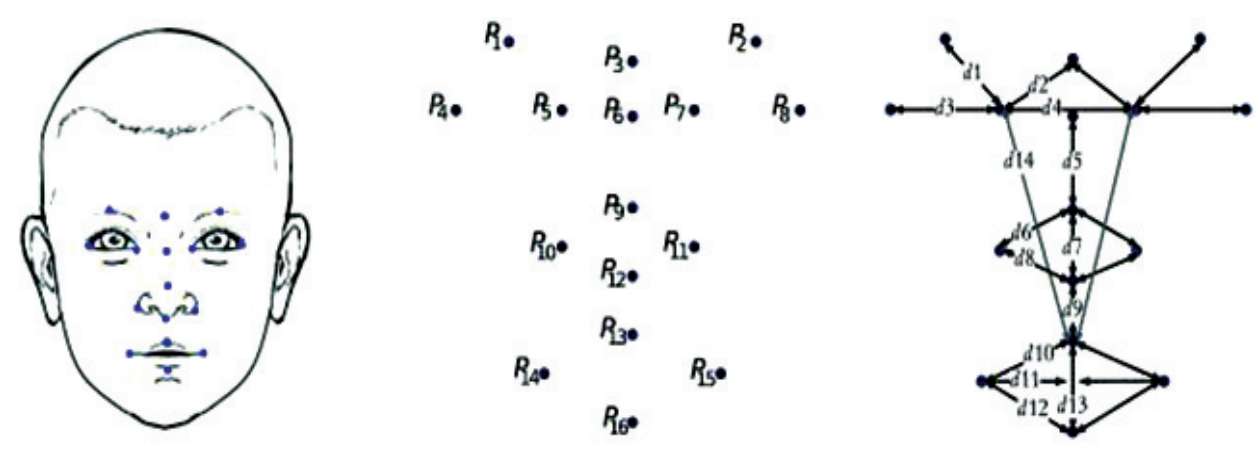

Figure 3. Localization of facial points

\section{RESULTS AND DISCUSSION}

Fiducial points are points that are used as points of reference or measure; hence determining the fiducial points is the elementary step to distinguish a face. Some of the main fiducial points are the eyes, lip edges, nose, and chin. In this study, some images from the Dartmouth Database of Children's Faces are selected, inclusive of males and female images that were showing very distinct facial expressions. Some images collected by Ferry et.al with DS are also admitted. The images were actually captured impulsively from unrelated angles, backgrounds, and distances, screening unreliable head poses, facial expressions, and occlusions. As the next step, the image is tested and verified and then compared to the solution with other 
prominent classifiers. The proposed methodology mainly composed of four stages: i) facial detection, ii) features extraction, iii) features reduction, and iv) classification. It can be remarked that from the study that as the number of iterations increased, there is a diminution in the error value. BPNN is used to train the classified parameters and MATLAB is the program utilized for training and testing. Figure 4 shows the performance of the AdaBoost algorithm and Table 2 depicts the evaluation of various methods for DS identification according to correctness rates (in percent).

Following, we compare the anticipated response of Ada-BPNN with other presented methods. The given system has enhanced performance than the later model. The accuracy of the planned technique is superior than the additional methods. This is the main purpose to use BP for parameter optimization and as a weak classifier; this system is hybrid with AdaBoost technology. Enhanced prediction accurateness has provided by a stronger classifier. The accuracy of the system is calculated using the expression,

$$
\text { Accuracy }=\frac{T P+T N}{T P+T N+F P+F N}
$$

For training purpose $70 \%$ of data is used, for testing $15 \%$, and for validation $15 \%$. For the training 7-1-1, the ANN structure is applied. For the prediction, the Ada-BPNN model uses the BPNN of feeble classifiers to compile to strong. The neural network toolbox in MATLAB is used to construct the models. The mean error rate of a strong classifier is $3.4 \%$ and for the weaker classifier, it is $5.6 \%$. The competence of the projected system is more eminent than the normal BP model and other existing examples. The best validation performance and the regression analysis are expressed in Figure 5 and Figure 6 (see in appendix) respectively.

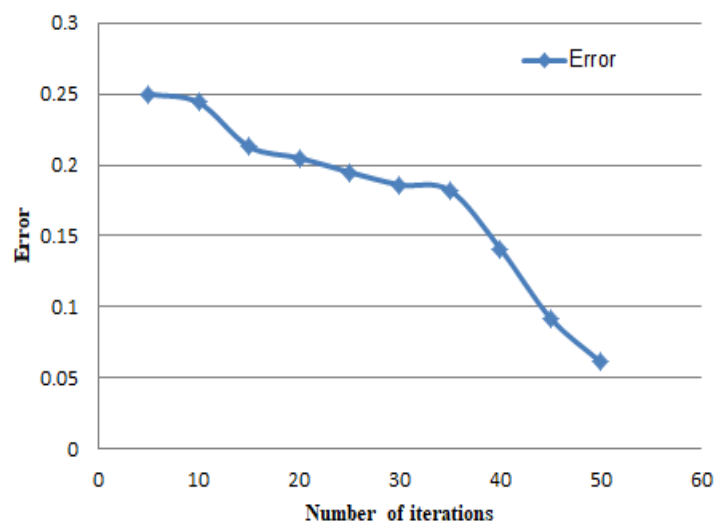

Figure 4. Adaboost performance

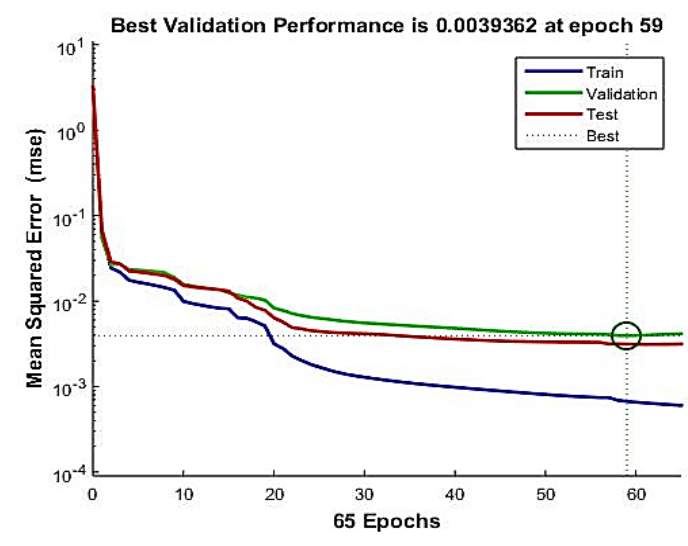

Figure 5. NN performance graph (using MATLAB)

Table 2. Accuracy comparison

\begin{tabular}{cccc}
\hline Access & Scheme & Number of images & Accuracy (\%) \\
\hline Zhao et al. [23] & SVM & 48 & 97.90 \\
Kruszka et al. $[24]$ & SVM & 65 & 94.30 \\
Burcin and Vasif [25] & LBPs & 107 & 95.30 \\
Erogul et al. [26] & EBGM & 86 & 68.70 \\
Proposed Model & Adaboost +BP & 82 & 98.67 \\
\hline
\end{tabular}

\section{CONCLUSION}

A feed-forward artificial neural network based on a boosting technique for the premature detection of DS is demonstrated. For this purpose-designed a single layer of hidden neurons and a single output neuron network. There is no proper treatment for Down syndrome; so its early detection is really indispensable and necessary. The proposed technique is based on facial identification and localization of facial points of the fetus. By computing the length between these points, the proposed algorithm predicts the chance for Down syndrome. Facial identification is performed with the help of AdaBoost and neural network training is performed with the help of MATLAB. Further, it is possible to increase the accuracy by increasing the number of training iterations and selecting more sensitive facial localization points. 


\section{APPENDIX}
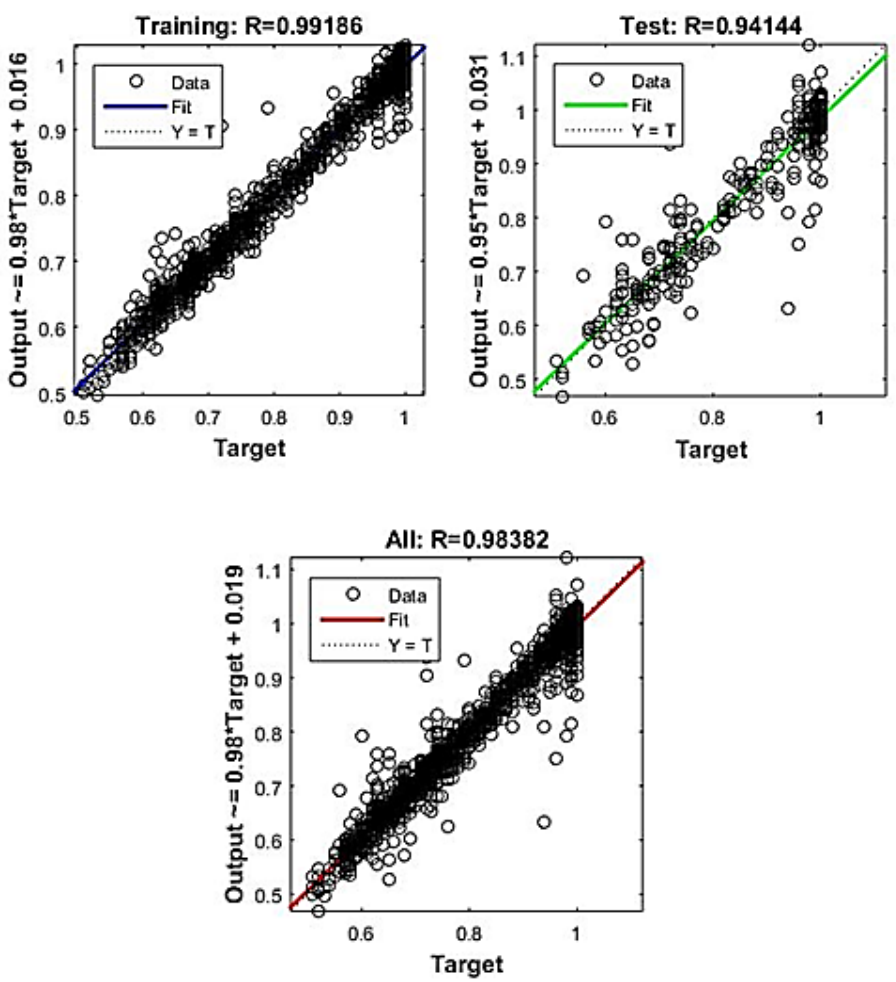

Figure 6. NN performance-regression analysis

\section{REFERENCES}

[1] B. H. Connolly, S. B. Morgan, F. F. Russell, and W. L. Fulliton, "A Longitudinal Study of Children with Down Syndrome Who Experienced Early Intervention Programming," Phys Ther, vol. 73, no. 3, pp. 170-179, 1993, doi: $10.1093 / \mathrm{ptj} / 73.3 .170$.

[2] A. Mittal, H. Gaur, and M. Mishra, "Detection of Down Syndrome Using Deep Facial Recognition," in B. Chaudhuri, M. Nakagawa, P. Khanna, and S. Kumar (Eds), Proceedings of 3rd International Conference on Computer Vision and Image Processing Advances in Intelligent Systems and Computing, vol. 1022, pp. 119-130, 2020, doi: 10.1007/978-981-32-9088-4_11.

[3] V. Dima, A. Ignat, and C. Rusu, "Identifying Down syndrome Cases by Combined Use of Face Recognition Methods," in V. Balas, L. Jain, and M. Balas (Eds), Soft Computing Applications Advances in Intelligent Systems and Computing, Springer, Cham, vol. 634, pp. 472-482, 2018, doi: 10.1007/978-3-319-62524-9_35.

[4] Q. Zhao, K. Rosenbaum, K. Okada, D. J. Zand, R. Sze, M. Summar et al., "Automated Down Syndrome Detection using Facial Photographs," 35th Annual International Conference of the IEEE EMBS, Osaka, Japan, Jul. 2013, pp. 3670-3673, doi: 10.1109/EMBC.2013.6610339.

[5] H. S. Loos, D. Wieczorek, R. P. Würtz, C. von der Malsburg, and B. Horsthemke, "Computer-based recognition of dysmorphic faces," European Journal of Human Genetics, vol. 11, no. 8, pp. 555-560, 2003, doi: 10.1038/sj.ejhg.5200997.

[6] S. Boehringer, M. Guenther, S. Sinigerova, R. P. Wurtz, B. Horsthemke, and D. Wieczorek, "Automated syndrome detection in a set of clinical facial photographs," American Journal of Medical Genetics Part A, vol. 155, no. 9, pp. 2161-2169, 2011, doi: 10.1002/ajmg.a.34157.

[7] S. Saraydemir, N. Taşpınar, O. Eroğul, H. Kayserili, and N. Dinçkan., "Down Syndrome Diagnosis Based on Gabor Wavelet Transform," Journal of Medical Systems, vol. 36, no. 5, pp. 3205-3213, 2012, doi: 10.1007/s10916011-9811-1.

[8] T. A. Anjit and S. Rishidas, "Identification of nasal bone for the early detection of Down syndrome using Back Propagation Neural Network," 2011 International Conference on Communications and Signal Processing, Calicut, 2011, pp. 136-140, doi: 10.1109/ICCSP.2011.5739286.

[9] C. Larose, P. Massac, Y. Hillion, J. P. Bernard, and Y. Ville, "Comparison of fetal nasal bone assessment by ultrasound at 11-14 weeks and by postmortem X-ray in trisomy 21: a prospective observational study," Ultrasound in Obstetrics and Gynecology, John Wiley \& Sons, Ltd, vol. 22, no. 1, pp. 27-30, 2003, doi: 10.1002/uog. 169 .

[10] K. O. Kagan, S. Cicero, I. Staboulidou, D. Wright, and K. H. Nicolaides, "'Petal nasal bone in screening for 
trisomies 21, 18 and 13 and Turner syndrome at LL- 13 weeks of gestation," Ultrasound in Obstetrics and Gynecology, John Wiley \& Sons, Ltd, vol. 33, pp. 259-264, 2004, doi: 10.1002/uog.6318.

[11] J. Sochman and J. Malas, "AdaBoost with totally corrective updates for fast face detection," Proceedings of Sixth IEEE International Conference on Automatic Face and Gesture Recognition, Seoul, South Korea, 2004, pp. 445-450, doi: 10.1109/AFGR.2004.1301573.

[12] C. S. Ram, "Recognizing Face Emotion of Down Syndrome Children using Viola Jone Technique," International Journal of Computer Science Trends and Technology (IJCST), vol. 7, no. 2, pp. 93-100, 2019.

[13] M. G. Krishna and A. Srinivasulu, "Face Detection System On AdaBoost Algorithm Using Haar Classifiers," International Journal of Modern Engineering Research, vol. 2, no. 5, pp. 3356-3560, 2012.

[14] J. Y. R. Cornejo, H. Pedrini, A. Machado-Lima, and F. D. L. dos Santos Nunes., "Down syndrome detection based on facial features using a geometric descriptor," Journal of medical imaging (Bellingham, Wash.), vol. 4, no. 4, 2017, Art. No. 044008, doi: 10.1117/1.JMI.4.4.044008.

[15] R. Lienhart, A. Kuranov, and P. Vadim, "Empirical analysis of detection cascades of boosted classifiers for rapid object detection," in Joint Pattern Recognition Symposium, DAGM 2003: Pattern Recognition, pp. 297-304, Sep. 2003, doi: 10.1007/978-3-540-45243-0_39.

[16] P. Viola and M. Jones, "Robust real-time objects detection," in International journal of computer vision, vol. 4, no. 34-47, 2001.

[17] S. P. Arjunan and M. C. Thomas, "A Review of Ultrasound Imaging Techniques for the Detection of Down Syndrome," IRBM, 2019. doi: 10.1016/j.irbm.2019.10.004.

[18] Y. Freund and R. E. Schapire, "A Short Introduction to Boosting," Journal of Japanese Society for Artificial Intelligence, vol. 14, no. 5, pp. 771-780, 1999.

[19] R. R. Chillarige, S. Distefano, and S. S. Rawat, "Advances in Computational Intelligence and Informatics," Lecture Notes in Networks and Systems, 2020, doi: 10.1007/978-981-15-3338-9.

[20] X. Liu, J. Bao, Y. Jiang, Z. Ke, and C. Wang, "College graduates' employment prediction based on Principal Component Analysis and the combined adaptive boosting and the backpropagation neural network algorithm," 2015 11th International Conference on Natural Computation (ICNC), 2015, pp. 1133-1137, doi: $10.1109 /$ icnc.2015.7378151.

[21] V. M. Joy and S. Krishnakumar, "Optimal Design of Power Scheduling using Artificial Neural Network in An Isolated Power System,” Int. J.of Pure and Applied Mathematics, vol. 118, no. 8, pp. 289-294, 2018.

[22] V. M. Joy and S. Krishnakumar, "Optimal design of adaptive power scheduling using modified ant colony optimization algorithm," International Journal of Electrical and Computer Engineering (IJECE), vol. 10, no. 1, pp. 738-745, 2020.

[23] Q. Zhao, K. Rosenbaum, R. Sze, D. Zand, M. Summar, and M. G. Linguraru, "Down syndrome detection from facial photographs using machine learning techniques," Medical Imaging 2013: Computer-Aided Diagnosis, 2013. doi: $10.1117 / 12.2007267$.

[24] P. Kruszka, A. R. Porras, A. K. Sobering, F. A. Ikolo, S. La Qua, V. Shotelersuk et al., "Down syndrome in diverse populations," American Journal of Medical Genetics Part A., vol. 173, no. 1, pp. 42-53, 2017, doi: 10.1002/ajmg. a. v173.1.

[25] Burçin K. and Vasif N. V., "Down syndrome recognition using local binary patterns and statistical evaluation of the system," Expert Syst. Appl. vol. 38, no. 7, pp. 8690-8695, 2011. doi: 10.1016/j.eswa.2011.01.076.

[26] O. Erogul, M. E. Sipahi, Y. Tunca and S. Vurucu, "Recognition of Down syndromes using image analysis," in 14th National Biomedical Engineering Meeting, pp. 1-4, 2009. doi: 10.1109/BIYOMUT.2009.5130322.

\section{BIOGRAPHIES OF AUTHORS}

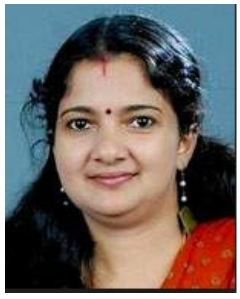

Vincy Devi V. K. is working as Assistant Professor in the department of Computer Applications, Sree Narayana Gurukulam College of Engineering, Kadayiruppu, Ernakulam. She has 12 years of teaching experience. She is doing her research in Medical Image processing. Her area of interest include Computer Networking, Cloud Computing and Bigdata Analysis.

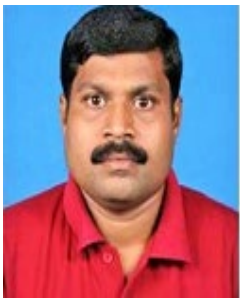

Rajesh R. is working as Associate Professor of Computer Science in the Department of Computer Science, CHRIST (Deemed to be University) Bangalore, India. Dr.Rajesh research interests are in the areas of Data Structures and Analysis of Algorithms. He has published 33 papers in various Journals and Conferences. Dr. Rajesh is also serving as Managing Editor, Lead Guest Editor, Associate Editor, Editorial board member and Technical Committee member of various National and International Conferences and Journals. He has received Veenus International Foundation's Outstanding Faculty award and Dewang Mehta Education Leadership Award to his credit. 